\title{
On the Cone of Nonnegative Circuits
}

\author{
Alan J. Hoffman ${ }^{1}$ and Carl W. Lee ${ }^{2 *}$ \\ ${ }^{1}$ IBM Thomas J. Watson Research Center, Yorktown Heights, NY 10598, USA \\ ${ }^{2}$ Department of Mathematics, University of Kentucky, Lexington, KY 40506, USA
}

\begin{abstract}
We discuss three equivalent formulations of a theorem of Seymour on nonnegative sums of circuits of a graph, and present a different (but not shorter) proof of Seymour's result.
\end{abstract}

\section{Introduction}

Let $G$ be a connected bridgeless graph. If $X \subseteq V(G), \partial(X)$ is the set of all edges $e$ with exactly one end in $X$. If $e \in \partial(X)$, the vector $h(X, e)$, with coordinates indexed by $E(G)$, is

$$
h(X, e)_{f}=\left\{\begin{aligned}
1 & \text { if } f \in \partial(X) \backslash\{e\} \\
-1 & \text { if } f=e \\
0 & \text { otherwise. }
\end{aligned}\right.
$$

A vector $x$ conforms to a vector $y$ if, for every $j, x_{j}>0$ implies $y_{j}>0$ and $x_{j}<0$ implies $y_{j}<0$. Denote by $M$ the $(0,1)$-matrix with rows corresponding to all circuits of $G$, columns to all edges of $G$, with

$$
M_{C e}= \begin{cases}1 & \text { if } e \in C \\ 0 & \text { otherwise }\end{cases}
$$

\footnotetext{
* Research supported, in part, by an IBM Postdoctoral Fellowship, a grant of the Alexander von Humboldt-Stiftung, and NSF grant DMS-8504050.
} 
Let $K(M)=\{z: M z \geq 0\}$. Thus $K(M)$ is the set of all edge flows (assignments of weights to edges) for which the total flow of every circuit is nonnegative. Note that $h(X, e) \in K(M)$ for all $X$ and $e$. For an edge flow $z$, set $N(z)=\left\{C:(M z)_{C}=0\right\}$, the set of circuits whose total flow is zero with respect to $z$. Such circuits will be called 0 -circuits.

This paper originated from an attempt to find a short proof of a remarkable theorem of Seymour [2] (Theorem 1 below), which is the undirected analog of Hoffman's circulation theorem for directed networks [1]. We wanted to establish Theorem 1 by proving an equivalent result (Theorem 2) about generators of $K(M)$. But that program seemed to require a third result (Theorem 3 ) which, although an easy consequence of Theorem 1 , is not so easy without it. Our discussion: Theorem $2 \Rightarrow$ Theorem $1 \Rightarrow$ Theorem $3 \Rightarrow$ Theorem 2 , together with a separate proof of a stronger result (Theorem 4) of which Theorem 3 is a corollary, yields a different proof of Theorem 1 , but not a shorter one.

Theorem 1. For any $c, d \in \mathbb{R}^{E(G)}$, the system of inequalities

$$
y \geq 0, \quad c^{\prime} \leq y^{\prime} M \leq d^{\prime}
$$

is consistent if and only if

$$
\begin{aligned}
& d \geq 0, \\
& d \geq c,
\end{aligned}
$$

and

$$
\text { for every } X \subseteq V(G) \text {, and every } e \in \partial(X), \quad c_{e} \leq \sum_{f \in \partial(X) \backslash\{e\}} d_{f}
$$

Note that $y$ above is a vector of flows on circuits (an assignment of weights to circuits). This is the analog to flow conservation in directed networks. Then (1.1) corresponds to lower and upper bounds on the cumulative arc flows.

Theorem 2. Every $z \in K(M)$ is a nonnegative sum of nonnegative vectors conforming to $z$ and vectors $h(X, e)$ conforming to $z$.

Theorem 3. If $z \in K(M)$ and $E(G)=\bigcup_{C \in N(z)} E(C)$, then if $v_{1}, v_{2} \in V(G)$, there is a path $P$ joining $v_{1}$ and $v_{2}$ such that $z(P)=\sum_{e \in P} z_{e} \leq 0$.

\section{Theorem $2 \Rightarrow$ Theorem 1}

The necessity of $(1.2 \mathrm{a})-(1.2 \mathrm{c})$ is obvious. To show they are sufficient, rewrite (1.1) as

$$
y^{\prime}[M ;-M] \leq\left[d^{\prime} ;-c^{\prime}\right], \quad y \geq 0
$$


By Farkas' theorem, (2.1) is consistent if and only if

$$
s \geq 0, \quad t \geq 0, \quad M(s-t) \geq 0 \quad \text { implies } \quad d^{\prime} s-c^{\prime} t \geq 0 .
$$

For any vector $z \in \mathbb{R}^{E(G)}$, let $z_{e}^{+}=z_{e}$ if $z_{e} \geq 0$, otherwise $z_{e}^{+}=0 ; z_{e}^{-}=-z_{e}$ if $z_{e} \leq 0$, otherwise $z_{e}^{-}=0$. Now consider $(s ; t)$ satisfying $s \geq 0, t \geq 0, M(s-t) \geq 0$. Then $(s ; t)=(q ; q)+\left(z^{+} ; z^{-}\right)$, where $q_{e}=\min \left(s_{e}, t_{e}\right)$ for each $e, z=s-t$, and $M z \geq 0$.

By $(1.2 \mathrm{~b}), d-c \geq 0$. But $s \geq 0, t \geq 0$ imply $q \geq 0$, so $d^{\prime} q-c^{\prime} q \geq 0$. By Theorem 2,

$$
\left(z^{+} ; z^{-}\right)=(p ; 0)+\sum k_{X}\left(h^{+}(X, e) ; h^{-}(X, e)\right),
$$

where $p \geq 0$, all $k_{X} \geq 0$. By (1.2a) $d^{\prime} p-c^{\prime} 0 \geq 0$, and by $(1.2 \mathrm{c}) d^{\prime} h^{+}(X, e)=$ $\sum_{f \in a(X) \backslash\{e\}} d_{f} \geq c_{e}=c^{\prime} h^{-}(X, e)$. This yields $d^{\prime} h^{+}(X, e)-c^{\prime} h^{-}(X, e) \geq 0$. So the implication (2.2) holds, and Theorem 1 is proved.

\section{Theorem $1 \Rightarrow$ Theorem 3}

Suppose $v_{1}$ and $v_{2}$ are ends of an edge $e \in E(G)$. By the hypothesis of Theorem 3 , there is a 0 -circuit containing $e$. If $z_{e} \leq 0$, the desired path is $e$. If $z_{e}>0$, the other edges of the 0 -circuit constitute the desired path.

Suppose $v_{1}$ and $v_{2}$ are not adjacent in $G$. Construct a graph $G^{*}$ by creating a new edge $f$ joining $v_{1}$ and $v_{2}$. We now describe vectors $c$ and $d$ for $G$. Let $c_{f}=1, d_{f}=\infty$. For all other edges $e \in E\left(G^{*}\right)$, let $c_{e}=d_{e}=$ the number of circuits $C \in N(z)$ containing $e$. It is easy to see that $(1.2 \mathrm{a})-(1.2 \mathrm{c})$ are satisfied. By the definition of $c$ and $d$, it follows from Theorem 1 applied to $G^{*}$ that in $G$ there exist paths $P_{1}, \ldots, P_{n}$ joining $v_{1}$ and $v_{2}$ and positive numbers $\alpha_{1}, \ldots, \alpha_{n}$, together with a (possibly empty) set of circuits $C_{1}, \ldots, C_{m}$ and associated positive numbers $\beta_{1}, \ldots, \beta_{m}$ such that, designating the $C$ th row of $M$ by $M_{C}$,

$$
\sum \alpha_{k} P_{k}+\sum \beta_{j} C_{j}=c=\sum_{C \in \mathcal{N}(z)} M_{C} .
$$

Note that in (3.1), as well as elsewhere in this paper, we may choose to abuse notation and identify paths, circuits or subgraphs with their incidence vectors. The above paths $P_{k}$ correspond to circuits in $G^{*}$ containing $f$. Because $c_{f}=1$ we know $n \geq 1$.

Take the inner product of $z$ with both the left- and right-hand sides of (3.1). Since $\left(C_{j}, z\right) \geq 0$ for all $j$ and $\left(M_{C}, z\right)=0$ for all $C \in N(z)$, it follows that $\left(P_{k}, z\right) \leq 0$ for at least one $k$.

\section{Theorem $3 \Rightarrow$ Theorem 2}

For any graph $G$ and any vector $z \in \mathbb{R}^{E(G)}$ with $M z \geq 0$, define $G_{z}$ as follows: $V\left(G_{z}\right)=V(G)$, and $v_{1}, v_{2}$ are adjacent in $G_{z}$ if and only if at least one of the following holds:

$$
z_{\left(v_{1}, v_{2}\right)} \leq 0
$$


or

there is a 0 -circuit containing $v_{1}$ and $v_{2}$.

It is easy to see that (4.1) and (4.2), using Theorem 3, imply:

if $v_{1}$ and $v_{2}$ are connected in $G_{z}$, there is a nonpositive path in $G$ joining $v_{1}$ and $v_{2}$.

For, let $G^{z}$ be obtained from $G$ by removing all edges that are in no 0-circuit of $z$. Then Theorem 3 applies to each component of $G^{z}$. The components of $G_{z}$ are obtained from the components of $G^{ \pm}$by (possibly) connecting some of the latter by negative edges. Hence (4.3) holds.

Recall that $z$ satisfies $M z \geq 0$. If $z \geq 0$, we are done, so assume $E(z)=\left\{e: z_{e}<0\right\}$ is not empty. We shall show there exist $X \subseteq V(G), f \in E(z) \cap \partial(X), t>0$ so that:

$$
\begin{gathered}
h(X, f) \text { conforms to } z ; \\
z^{*}=z-t h(X, f) \text { conforms to } z \text {, and satisfies } M z^{*} \geq 0 ; \\
\operatorname{supp} z^{*} \subseteq \operatorname{supp} z ; \\
N(z) \subseteq N\left(z^{*}\right) ;
\end{gathered}
$$

with at least one of the inclusions in (4.6) and (4.7) proper. Clearly, these stipulations on $z^{*}$ will suffice to prove Theorem 2 .

For each $e=\left(v_{i}, v_{j}\right) \in E(z)$, consider the graph $(G-e)_{z}$ defined above, and let $K_{i z}^{e}$ be the set of vertices of the component containing $v_{i}$ in that graph. By (4.3),

$$
K_{i z}^{e} \neq K_{j z}^{e}
$$

otherwise we would have a nonpositive path joining $v_{i}$ and $v_{j}$ which, together with $e$, would produce a negative circuit. Of all possible $K_{i z}^{e}$ which could be so constructed, choose one with the fewest number of vertices, say $K_{1 z}^{f}$. We first show

$$
\text { if } e=\left(v_{i}, v_{j}\right) \in E(G) \text { and } v_{i}, v_{j} \in K_{1 z}^{f} \text {, then } z_{e} \geq 0
$$

Assume otherwise. Let $P$ be the $\left(v_{1}, v_{i}\right)$-path in $(G-f)_{z} \subseteq G_{z}$ whose existence is guaranteed by $v_{i} \in K_{i z}^{f}$. Suppose $v_{1} \notin K_{1 z}^{e}$. Let $v_{k}$ be any vertex of $K_{i z}^{e}$ and $Q$ be a $\left(v_{i}, v_{k}\right)$-path in $(G-e)_{z} \subseteq G_{z}$. If $Q \notin(G-f)_{z}$ then $Q$ contains $f$ or some edge derived via (4.2) from a 0 -circuit containing $f$. In this case we can see that $v_{1}$ can also be reached from $v_{i}$ in $(G-e)_{z}$, contradicting $v_{1} \notin K_{i z}^{e}$. Hence $Q \subseteq(G-f)_{z}$. But then we can get from $v_{1}$ to $v_{k}$ in $(G-f)_{z}$ by first following $P$ and then following $Q$. Thus $v_{k} \in K_{1 z}^{f}$ and $K_{i z}^{e}$ is properly contained in $K_{1 z}^{f}$, contradicting the minimality of $K_{1 z}^{f}$. Therefore $v_{1} \in K_{i z}^{e}$. The same reasoning shows that $v_{1} \in K_{j z}^{e}$. But this means $K_{i z}^{e}=K_{j z}^{e}$, violating (4.8). So (4.9) is true. 

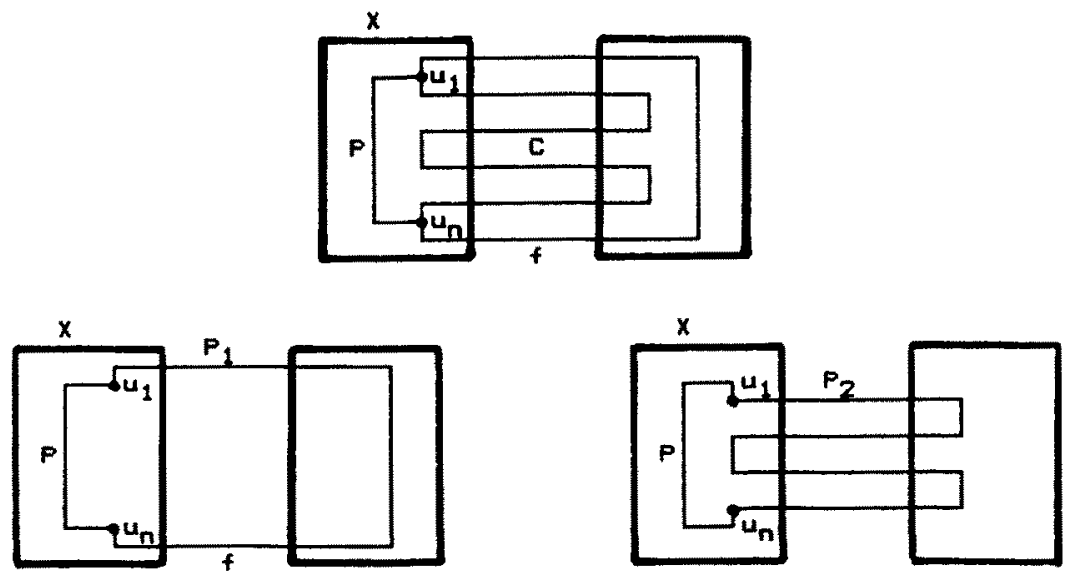

Fig. 1

Next, let $X=K_{1 z}^{f}$. The vector $h(X, f)$ satisfies (4.4), by (4.1). We now claim

$$
N(z) \subseteq N(h(X, f))
$$

Since $N\left(z^{*}\right)$ differs from $N(z)$ only on circuits crossing $X,(4.10)$ would imply that (4.7) is true.

To show (4.10) it is sufficient to show that $C \in N(z)$ implies that $C$ either misses $\partial(X)$ or intersects $\partial(X)$ in $f$ and exactly one (positive) edge. If $C$ intersects $\partial(X)$, but does not contain $f$, then we have a contradiction of (4.2). Further, by (4.1), every edge in $\partial(X) \backslash\{f\}$ is positive. Suppose $C$ contains more than one (positive) edge of $\partial(x)$. From (4.3) and (4.9), any two vertices of $C$ in $X$ can be connected by a path of 0-edges. It follows that, if $C$ contains more than one positive edge of $\partial(X)$, there is a path of 0 -edges, all vertices of which are in $X$, say $P=\left(u_{1}, u_{2}\right),\left(u_{2}, u_{3}\right), \ldots,\left(u_{n-1}, u_{n}\right)$, where $u_{1}$ and $u_{n}$ are in $C$, all others are not, and $C$ is the mod 2 sum of circuits $P+P_{1}$ and $P+P_{2}\left(P_{1}\right.$ and $P_{2}$ being paths whose union is $C$ ), and $P+P_{1}, P+P_{2}$ both contain edges of $\partial(X)$. (See Fig. 1.) But $\sum_{e \in P+P_{1}} z_{e} \geq 0$ and $\sum_{e \in P+P_{2}} z_{e} \geq 0$. Since $P$ is a 0 -path, and $C$ is a 0 -circuit, it follows that $P+P_{1}$ and $P+P_{2}$ are both 0 -circuits. But only one can contain $f$, so the other violates (4.2).

Let $t$ be the largest positive number such that $z^{*}$ satisfies (4.5). By (4.4) and (4.10), such a positive number exists, and at least one of (4.6) and (4.7) is proper inclusion.

\section{Proof of Theorem 3}

Theorem 3 follows as a corollary of the following stronger result:

Theorem 4. If $z \in K(M)$ and $E(G)=\bigcup_{C \in N(z)} E(C)$, then if $v_{1}, v_{2} \in V(G)$ there 
is a pair $P_{1}, P_{2}$ of $\left(v_{1}, v_{2}\right)$-paths and a (possibly empty) set of circuits $S$ such that (as incidence vectors)

$$
P_{1}+P_{2}+\sum_{C \in S} C=\sum_{C \in N(z)} M_{C}
$$

Once we have established Theorem 4, we can take the inner product of $z$ with both sides of (5.1). Then, just as in Section 3 , since $(C, z) \geq 0$ for all $C \in S$ and $\left(M_{C}, z\right)=0$ for all $C \in N(z)$, it follows that at least one of the two paths is nonpositive. Hence Theorem 3 holds.

Proof. Consider all subsets $T \subseteq N(z)$ for which $v_{1}$ and $v_{2}$ are connected in the subgraph $\bar{G}$ with edge set $E(\bar{G})=\bigcup_{C \in T} E(C)$. Determine the minimum of $|E(\bar{G})|$ over all such subsets $T$. Now, of those subsets $T$ which achieve this minimum, select one containing the fewest number of circuits, say $\left\{C_{1}, \ldots, C_{n}\right\}$.

If $n=1$ then $v_{1}$ and $v_{2}$ are contained in a common 0 -circuit. In this case we choose $P_{1}$ and $P_{2}$ to be the two $\left(v_{1}, v_{2}\right)$-paths determined by this circuit and $S$ to be all of the other 0 -circuits of $G$. Then (5.1) holds, and we are done. So assume that $n>1$.

From the minimality of $n$ we may assume (after appropriate relabeling if necessary) that

$$
v_{1} \in V\left(C_{1}\right) \backslash V\left(C_{2}\right), \quad v_{2} \in V\left(C_{n}\right) \backslash V\left(C_{n-1}\right),
$$

and

$$
V\left(C_{i}\right) \cap V\left(C_{j}\right) \neq \varnothing \text { if and only if }|i-j|=0 \text { or } 1 \text { (and hence no }
$$
vertex of $\bar{G}$ is in more than two of the $C_{i}$ ).

Suppose there is a vertex $v$ of $\bar{G}$ of degree larger than three. Then $v$ must be common to $C_{i}$ and $C_{i+1}$ for some $i$, and by (5.2) must in fact be of degree four. Reduce the degree of $v$ by splitting it, introducing a new edge with weight zero common to both $C_{i}$ and $C_{i+1}$. This splitting operation cannot create any negative circuit. For suppose $C$ is a circuit in the new graph. If the new edge is shrunk, $C$ will become either one or two nonnegative circuits whose total weight equals that of $C$. This argument shows also that if $C$ is a 0 -circuit in the new graph, the resulting circuits must be 0 -circuits in the shrunken graph.

Repeating the splitting procedure with other vertices, if necessary, we may assume

every vertex of $\bar{G}$ is of degree two or three.

As one consequence of the above assumptions, $C_{i}$ and $C_{i+1}$ share at least one edge, $1 \leq i \leq n-1$. 
Define the graphs

$$
\begin{aligned}
& G_{j}=\bigcup_{i=1}^{j} C_{i}, \quad j=1, \ldots, n, \\
& F_{j}= \begin{cases}C_{j} \cap C_{j+1}, & j=1, \ldots, n-1, \\
\left\{v_{2}\right\}, & j=n,\end{cases} \\
& D_{j}= \begin{cases}\varnothing, & j=0, \\
\bigcup_{i=1}^{j} F_{i}, & j=1, \ldots, n .\end{cases}
\end{aligned}
$$

Let $G_{j}^{(e)}$ be the graph obtained from $G_{j}$ by duplicating the edges of $G_{j}$ that are in $D_{j-1}, j=1, \ldots, n$; let $G_{j}^{(d)}$ be the subgraph of $G_{j}$ with edge set $E\left(G_{j}\right) \backslash E\left(D_{j-1}\right)$, $j=1, \ldots, n$; and let $B_{j}$ be the component of $G_{j}^{(d)}$ that contains $v_{1}, j=1, \ldots, n$. Note that $G_{j}^{(e)}$ is Eulerian and that $G_{j}^{(d)}$ is a vertex-disjoint union of circuits. Observe also that $z\left(G_{j}^{(e)}\right)=\sum_{i=1}^{j} z\left(C_{i}\right)=0$.

Now let $x_{0}=v_{1}$ and $x_{1}$ be the last vertex of $F_{1}$ encountered while traversing $C_{1}$ from $v_{1}$ to $v_{1}$. Define $R_{1}$ to be the $\left(x_{0}, x_{1}\right)$-path whose edges are contained in $E\left(B_{1}\right) \backslash E\left(C_{2}\right)=E\left(C_{1}\right) \backslash E\left(C_{2}\right)$, and $H_{1}$ to be the other $\left(x_{0}, x_{1}\right)$-path contained in $C_{1}$. Then the following three conditions hold for $i=1$ :

$$
x_{i} \text { is a vertex of } F_{i} \text { of degree three in } \bar{G} \text {, }
$$

$R_{i}$ is an $\left(x_{0}, x_{i}\right)$-path whose edges are contained in $E\left(B_{i}\right) \backslash E\left(C_{i+1}\right)$,

and

$H_{i}$ is a vertex-disjoint union of an $\left(x_{0}, x_{i}\right)$-path and (possibly) some circuits, such that $D_{i} \subseteq H_{i} \subseteq G_{i}$.

Assume (5.5)-(5.7) hold for $i=k$, for some $1 \leq k \leq n-1$. Note that $R_{k} \subseteq B_{k+1}$. Define $Q_{k+1}$ to be the $\left(x_{0}, x_{k}\right)$-path with edge set $E\left(B_{k+1}\right) \backslash E\left(R_{k}\right)$. Now $Q_{k+1}$ contains at least one edge $f$ in $E\left(C_{k+1}\right) \backslash E\left(C_{k}\right)$, one of the three edges incident to $x_{k}$. Observe that (5.3) implies that $f$ cannot be in $C_{k+2}$.

There are two cases to consider (see Figs. 2 and 3):

$$
F_{k+1} \notin Q_{k+1}
$$

or

$$
F_{k+1} \subseteq Q_{k+1}
$$

Case (5.8). Consider $G_{k+1}^{\prime}$, the $\bmod 2$ sum of $H_{k}$ and $Q_{k+1}$, a subgraph of $G_{k+1}$. From (5.8) we have $F_{k+1} \notin G_{k+1}^{\prime}$. Note that $G_{k+1}^{\prime}$ contains $f$, since $Q_{k+1}$ does and $H_{k} \subseteq G_{k}$ so $H_{k}$ contains no edge in $E\left(C_{k+1}\right) \backslash E\left(C_{k}\right)$. Let $G_{k+1}^{\prime \prime}$ be the subgraph of $G_{k+1}^{(e)}$ with edge set $E\left(G_{k+1}^{(e)}\right) \backslash E\left(G_{k+1}^{\prime}\right)$, regarding $G_{k+1}^{\prime}$ as a subgraph of $G_{k+1}^{(e)}$. 


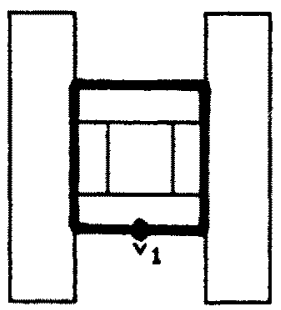

$\mathbf{c}_{1}$

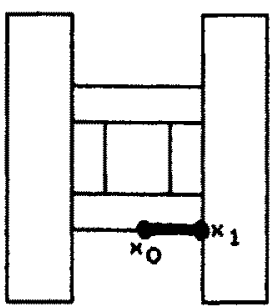

$\mathbf{R}_{\mathbf{1}}$

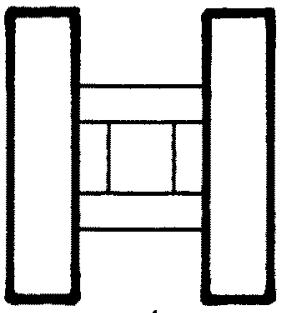

$\mathbf{E}_{2}^{\prime}$

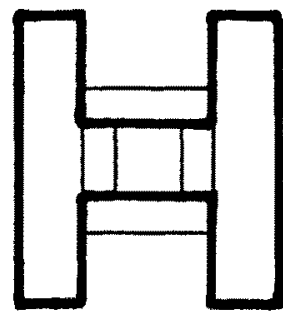

$\mathrm{c}_{2}$

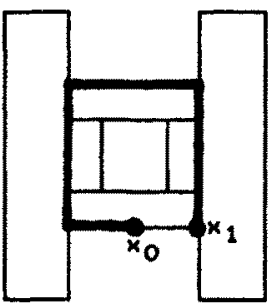

$H_{1}$

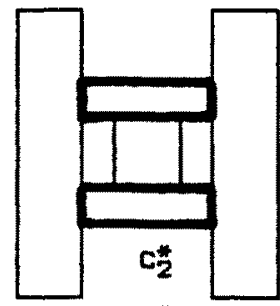

$\mathrm{G}_{2}^{\prime \prime}$

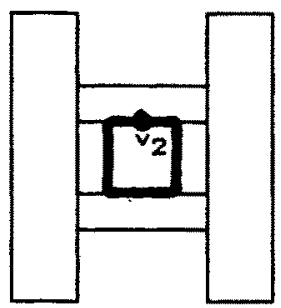

$\mathrm{C}_{3}$

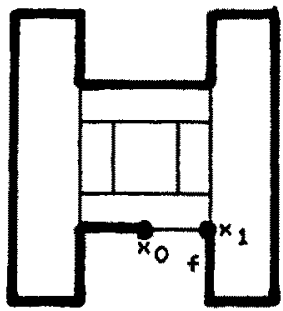

$\mathbf{\theta}_{2}$

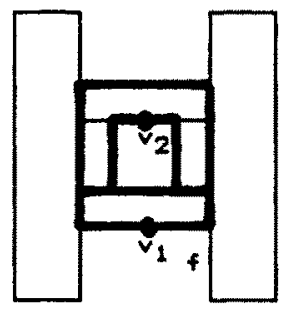

H

Fig. 2. Case (5.8).

For every duplicated edge in $G_{k+1}^{(e)}$, one edge of the pair is in $G_{k+1}^{\prime}$, while the other is in $G_{k+1}^{\prime \prime}$. This is true because $D_{k} \subseteq G_{k+1}^{\prime}$, since $D_{k} \subseteq H_{k}$ and no edge of $D_{k}$ is in $B_{k+1}$. Also, every vertex of $G_{k+1}^{\prime}$ is of even degree, as is every vertex of $G_{k+1}^{\prime \prime}$. Thus, both $G_{k+1}^{\prime}$ and $G_{k+1}^{\prime \prime}$ are vertex-disjoint unions of circuits of $G_{k+1}^{(e)}$, and in fact of circuits of $G_{k+1}$.

Then $z\left(G_{k+1}^{\prime}\right) \geq 0, \quad z\left(G_{k+1}^{\prime \prime}\right) \geq 0$ and $z\left(G_{k+1}^{\prime}\right)+z\left(G_{k+1}^{\prime \prime}\right)=z\left(G_{k+1}^{(e)}\right)=0$ force $z,\left(G_{k+1}^{\prime \prime}\right)=0$, and in fact we have $G_{k+1}^{\prime \prime}$ written as a vertex-disjoint union of 0 -circuits. By (5.8) there is a circuit $C_{k+1}^{*}$ of $G_{k+1}^{\prime \prime}$ that contains a vertex of $F_{k+1}$. Now $C_{k+1}^{*}$ also contains at least one edge of $C_{k}$, otherwise $(5.3)$ forces $E\left(C_{k+1}^{*}\right) \subseteq$ $E\left(C_{k+1}\right) \backslash E\left(C_{k}\right)$, which is impossible. However, we know also that $C_{k+1}^{*} \neq C_{k+1}$, because $C_{k+1}^{*}$ cannot contain $f$.

Consider the graph $H=C_{1} \cup \cdots \cup C_{k} \cup C_{k+1}^{*} \cup C_{k+2} \cup \cdots \cup C_{n}$, a proper subgraph of $\bar{G}$ since it does not contain $f$. The vertices $v_{1}$ and $v_{2}$ are connected in this graph. Start with $\bar{G}$, take all of the edges that were introduced by splitting vertices near the beginning of the proof, and shrink them. Do the same thing with $H$. Note that $f$ is contained in only one of the $C_{i}$ and hence is not one of those edges to be shrunk. The resulting shrunken $H$ will then be a proper subgraph 


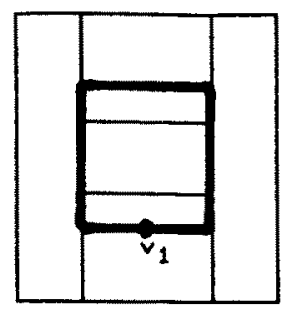

$c_{1}$

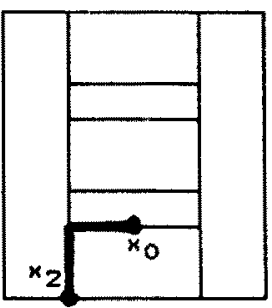

$\mathbf{R}_{\mathbf{2}}$

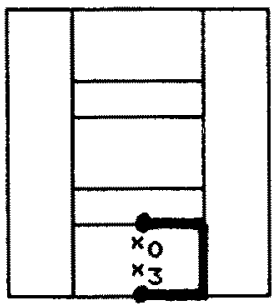

$\mathrm{R}_{3}$

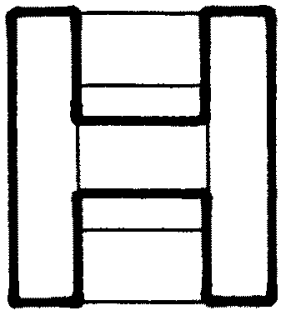

$\mathrm{C}_{2}$

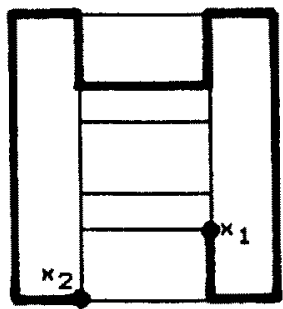

$\mathbf{P}_{\mathbf{2}}$

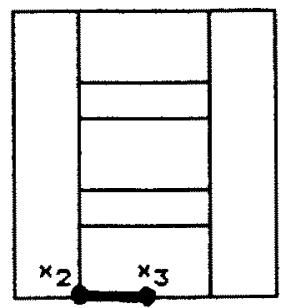

$\mathrm{P}_{3}$

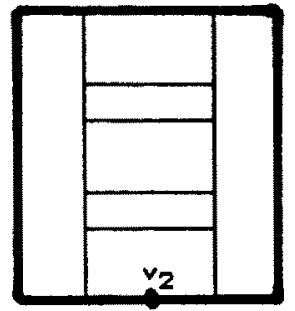

$\mathrm{c}_{3}$

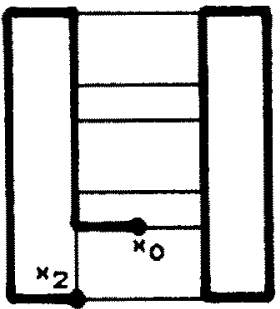

$\mathrm{H}_{2}$
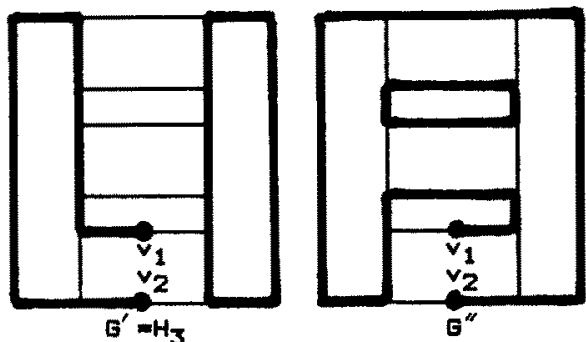

Fig. 3. Case (5.9).

of the shrunken $\bar{G}$. Every edge of $H$ is contained in a 0 -circuit of $H$ and $v_{1}, v_{2}$ are connected in $H$. Yet $|E(H)|<|E(\bar{G})|$, contradicting our initial assumption about the minimality of $|E(\bar{G})|$. Therefore case (5.8) cannot occur.

Case (5.9). Let $x_{k+1}$ be the first vertex of $F_{k+1}$ encountered while traveling from $x_{0}$ to $x_{k}$ in $Q_{k+1}$. Define $R_{k+1}$ to be the portion of the above path from $x_{0}$ to $x_{k+1}$, $P_{k+1}$ to be the portion of the above path from $x_{k+1}$ to $x_{k}$, and $H_{k+1}$ to be the mod 2 sum of $H_{k}$ and $P_{k+1}$, a subgraph of $G_{k+1}$. By (5.9), $F_{k+1} \subseteq P_{k+1}$, implying that $D_{k+1} \subseteq H_{k+1}$, so (5.5)-(5.7) hold for $i=k+1$.

Since case (5.8) is never encountered, we will eventually construct $H_{n}$. First note that $x_{n}=v_{2}$. Let $G^{\prime}=H_{n}$, and $G^{\prime \prime}$ be the subgraph of $G_{n}^{(e)}$ with edge set $E\left(G_{n}^{(e)}\right) \backslash E\left(G^{\prime}\right)$, regarding $G^{\prime}$ as a subgraph of $G_{n}^{(e)}$. For every duplicated edge in $G_{n}^{(e)}$, one edge of the pair is in $G^{\prime}$, while the other is in $G^{\prime \prime}$, because $D_{n} \subseteq H_{n}$. Also, every vertex of $G^{\prime}$ is of even degree, except $x_{0}=v_{1}$ and $x_{n}=v_{2}$, which are of degree one, and the same is true of $G^{\prime \prime}$. Thus $G^{\prime}$ and $G^{\prime \prime}$ each consists of a $\left(v_{1}, v_{2}\right)$-path, $P^{\prime}$ and $P^{\prime \prime}$, respectively, and a vertex-disjoint union of circuits of $G_{n}^{(e)}$, and in fact of circuits of $G_{n}=\tilde{G}$. 
We now have (as incidence vectors) $P^{\prime}+P^{\prime \prime}+\sum_{C \in S^{\prime}} C+\sum_{C \in S^{\prime \prime}} C=\sum_{i=1}^{n} C_{i}$. Shrink those edges that were used to split vertices near the beginning of the proof. The result will be two $\left(v_{1}, v_{2}\right)$-paths $P_{1}, P_{2}$ and a set $T$ of circuits such that $P_{1}+P_{2}+\sum_{C \in T} C=\sum_{i=1}^{n} C_{i}$ in this shrunken $\bar{G}$. (Note that each path will shrink into a path, together with (possibly) some circuits.) Finally, we arrive at the desired set $S$ of 0 -circuits by adding to $T$ all 0 -circuits not in $\left\{C_{1}, \ldots, C_{n}\right\}$. Then (5.1) holds.

\subsection{Examples}

In Fig. 2 we have three circuits $C_{1}, C_{2}$, and $C_{3}$. Tracing $C_{1}$ clockwise from $x_{0}=v_{1}$ to $v_{1}$ the last vertex of $C_{2}$ we encounter is $x_{1}$. The path $R_{1}$ is the $\left(x_{0}, x_{1}\right)$-path lying only in $C_{1} ; H_{1}$ is the other $\left(x_{0}, x_{1}\right)$-path. We now construct $Q_{2}$ by leaving $x_{0}$ in the opposite direction of $R_{1}$, staying on edges lying in exactly one of $C_{1}$ or $C_{2}$, until we arrive at $x_{1}$. Observe that $F_{2}$, the intersection of $C_{2}$ and $C_{3}$ is not contained in $Q_{2}$ (in fact, it is missed entirely). Thus we are in case (5.8). We let $G_{2}^{\prime}$ be the mod 2 sum of $H_{1}$ and $Q_{2}$, and choose $G_{2}^{\prime \prime}$ to be the graph having all edges that are in $C_{1} \cap C_{2}$ as well as those edges of $C_{1}$ and $C_{2}$ that are missed by $G_{2}^{\prime}$. Pick a circuit $C_{2}^{*}$ of $G_{2}^{\prime \prime}$ that meets $F_{2}$ (either will do). Then $H=C_{1} \cup C_{2}^{*} \cup$ $C_{3}$ is a union of 0 -circuits in which $v_{1}$ and $v_{2}$ are connected, and $H$ has fewer edges than $\bar{G}=C_{1} \cup C_{2} \cup C_{3}$.

If, however, we keep $C_{1}$ and $C_{2}$ as in Fig. 2 but alter $C_{3}$ and $v_{2}$ to get Fig. 3, we will construct the same $R_{1}, H_{1}$, and $Q_{2}$ as before. But now $F_{2}$ is completely contained in $Q_{2}$ so we proceed as in case (5.9). Following $Q_{2}$ from $x_{0}$ to $x_{1}$, the first vertex of $C_{3}$ we encounter is $x_{2}$. The part of $Q_{2}$ between $x_{0}$ and $x_{2}$ is $R_{2}$; the rest of $Q_{2}$ is $P_{2}$. We set $H_{2}$ to be the mod 2 sum of $H_{1}$ and $P_{2}$. Construct $Q_{3}$ by leaving $x_{0}$ in the opposite direction of $R_{2}$, staying on edges lying in exactly one of $C_{1}, C_{2}$ or $C_{3}$, until we reach $x_{2}$. Note that $F_{3}$, the point $v_{2}$ itself which we now call $x_{3}$, is contained in $Q_{3}$. So again we are in case (5.9). The part of $Q_{3}$ between $x_{0}$ and $x_{3}$ is $R_{3}$; the rest of $Q_{3}$ is $P_{3}$. The mod 2 sum of $H_{2}$ and $P_{3}$ is $H_{3}$, which is also $G^{\prime}$. We get $G^{\prime \prime}$ by choosing all edges of $C_{1} \cup C_{2} \cup C_{3}$ that lie in exactly two of these three circuits, together with all other edges missed by $G^{\prime}$. Each of $G^{\prime}$ and $G^{\prime \prime}$ has a $\left(v_{1}, v_{2}\right)$-path, and at least one of these must be nonpositive.

\section{Remarks}

One of the referees kindly provided the following information: A. Sebö (Budapest) independently arrived at Theorem 3 through his work on $t$-joins, which are closely related to sums of circuits. Among the results he obtained is that under the conditions of Theorem 3 (or the slightly weaker condition that every positive edge is in a 0 -circuit and all circuits are nonnegative) the distance $d(x, y)$ between any two vertices $x, y$ is nonpositive, the relation $x \sim y$ if and only if $d(x, y)=0$ 
is an equivalence relation, and that properties of the equivalence classes can be used to obtain a Kotzig-type theorem for $t$-joins.

\section{Acknowledgments}

We are grateful to Don Coppersmith, George Dantzig, and Richard Stone for helpful comments about this work. Our greatest debt is to Heinz Gröflin who found an error in an early proof of Theorem 3 .

\section{References}

1. A. J. Hoffman, Some recent applications of the theory of linear inequalities to extremal combinatorial analysis, Proc. Sympos. Appl. Math. 10 (1960). See also L. R. Ford, Jr. and D. R. Fulkerson, Flows in Networks, 51, Princeton University Press, Princeton, 1974.

2. P. D. Seymour, Sums of circuits, in Graph Theory and Related Topics, 341-355 (J. A. Bondy and U. S. R. Murty, eds.), Academic Press, New York. 1979.

Received May 29, 1985, and in revised form December 16, 1985. 